\title{
Diboson production at the LHC with the CMS detector
}

\author{
Matthieu Marionneau* \\ SPP/IRFU CEA-Saclay \\ E-mail: matthieu.pierre.marionneaudcern.ch
}

The measurement of diboson production cross sections for a center-of-mass energy of $7 \mathrm{TeV}$ is presented. Luminosities used are $1.1 \mathrm{fb}^{-1}$ from 2011 data for $\mathrm{ZZ}$, WZ and WW processes and $36 \mathrm{pb}^{-1}$ from 2010 data for $\mathrm{W} \gamma$ and $\mathrm{Z} \gamma$ final states. Cross sections are measured to $\sigma(\mathrm{pp} \rightarrow \mathrm{ZZ}+\mathrm{X})=3.8_{-1.2}^{+1.5}$ (stat.) \pm 0.2 (syst.) \pm 0.2 (lumi.) $\mathrm{pb}, \sigma(\mathrm{pp} \rightarrow \mathrm{WZ}+\mathrm{X})=17.0 \pm$ 2.4 (stat.) \pm 1.1 (syst.) \pm 1.0 (lumi.) pb and $\sigma(\mathrm{pp} \rightarrow \mathrm{WW}+\mathrm{X})=55.3 \pm 3.3$ (stat.) \pm 6.9 (syst.) \pm 3.3 (lumi.) pb. The production cross sections of processes including photons in the final state, $\mathrm{W} \gamma$ and $\mathrm{Z} \gamma$ processes are given. Results obtained with 2010 data are also interpreted in term of anomalous triple gauge couplings.

The 2011 Europhysics Conference on High Energy Physics, EPS-HEP 2011,

July 21-27, 2011

Grenoble, Rhône-Alpes, France

* On behalf of the CMS collaboration 


\section{Diboson physics at LHC}

The study of diboson production at LHC represents a fundamental test of the standard model (SM) with the capacity to probe the self-interaction between gauge bosons, a direct consequence of the non-abelian nature of the electroweak sector of the SM. Values of couplings are given by the $\mathrm{SM}$, and the observation of deviations to the predictions is an indication of new physics. These deviations imply an increase of the production cross sections and possible distortions of distributions of several observables, such as the transverse momentum spectrum of one of the two bosons in the final state.

In this proceeding we present the diboson cross section measurements in fully leptonic channels made by the CMS collaboration in 2010 and 2011, using pp collision data produced at $\sqrt{s}=$ $7 \mathrm{TeV}$. First, results for ZZ, WZ and WW final states [1] and obtained using $1.1 \mathrm{fb}^{-1}$ are given. $\mathrm{W} \gamma$ and $\mathrm{Z} \gamma$ results from 2010 analyses $\left(36 \mathrm{pb}^{-1}\right)$ are also presented [2]. In the last section are described the measurements of anomalous triple gauge couplings with 2010 data [2,3]. We do not describe the CMS detector in detail. A complete description of the detector and informations about objects reconstruction can be found in [4].

\section{Measurement of $\mathrm{ZZ}$ production cross section}

The $\mathrm{ZZ}$ production cross section is measured using the four charged-lepton decay channel $\mathrm{ZZ} \rightarrow l^{ \pm} l^{\mp} l^{\prime \pm} l^{\prime \mp}$, with $l=e, \mu$ and $l^{\prime}=e, \mu, \tau$. The first $\mathrm{Z}$ boson candidate is reconstructed using two well, identified, opposite sign, isolated leptons of same flavors, with a transverse momentum $p_{T}$ of 20(10) GeV for the leading (trailing) lepton. Other leptons from the second $\mathrm{Z}$ boson are required to have $p_{T}>7(5) \mathrm{GeV}$ for $e e / \mu \mu$ channels and $p_{T}(\tau)>15 \mathrm{GeV}$ with additionnal restrictions on $p_{T}$ of the decay product of the $\tau: p_{T}>10 \mathrm{GeV}$ for $e e / \mu \mu$ decays and $p_{T}$ (jet) $>20 \mathrm{GeV}$ for hadronic decays. Finally, a cut is applied on the lepton impact parameter to reduce background from heavy flavor decays.

Events with $\mathrm{Z}$ bosons decaying into electrons and muons are selected by requiring the dilepton invariant mass to be between 60 and $120 \mathrm{GeV}$. When final state includes $\tau$, the visible mass of the $\mathrm{Z}$ boson formed by the decay products of $\tau$ must be in the range $30<M_{Z}^{\text {visible }}<80 \mathrm{GeV}$.

Few experimental backgrounds are still present, due to the desintegration of heavy hadrons in leptons $(\mathrm{Z}+b$ jets, $t \bar{t})$, misidentified leptons from jets $(\mathrm{Z}+$ jets $)$ and the $\mathrm{WZ}$ background for final states including $\tau$. These backgrounds are measured using dedicated control samples. The $t \bar{t}$ and $\mathrm{Z}+b$ jets backgrounds are estimated using a control sample defined by the inversion of the impact parameter selection. The misidentified lepton rate is estimated from a control sample defined by reverting the identification/isolation criteria applied on leptons. $\tau$ misidentification rate is estimated from a sample enriched in misidentified lepton, obtained by relaxing isolation and charge requirement. The number of selected events in data, SM predictions and main background contributions is indicated in Table 2.

The sources of systematic uncertainties are related to lepton reconstruction, triggering and energy scale. These uncertainties are evaluated using data. The theoretical uncertainty linked to 


\begin{tabular}{cccc}
\hline \hline Final state & $\mathrm{N}_{\text {obs. }}$ & $\mathrm{N}_{\text {estim. }}^{\text {back. }}$ & $\mathrm{N}_{\text {exp. }}^{Z Z}$ \\
\hline $4 \mu$ & 2 & $0.004 \pm 0.004$ & $3.7 \pm 0.4$ \\
$4 e$ & 0 & $0.14 \pm 0.06$ & $2.5 \pm 0.2$ \\
$2 e 2 \mu$ & 6 & $0.15 \pm 0.06$ & $6.3 \pm 0.6$ \\
$212 \tau$ & 1 & $0.8 \pm 0.1$ & $1.4 \pm 0.1$ \\
\hline \hline
\end{tabular}

Table 1: Number of selected event in data and standard model prodiction for backgrounds and signal, for the $\mathrm{ZZ}$ analysis.

\begin{tabular}{l|r}
\hline \hline source & uncertainty \\
\hline trigger & $1.5 \%$ \\
lepton identification & $3 \%$ \\
lepton isolation & $2 \%$ \\
lepton energy scale & $1 \%$ \\
$\tau$ reconstruction & $6 \%$ \\
$\tau$ energy scale & $3 \%$ \\
\hline \hline
\end{tabular}

Table 2: Main experimental systematic uncertainties on $\mathrm{ZZ}$ cross section measurement.

the detector acceptance is estimated to $3.6 \%$ in average. Uncertainty on luminosity is $6 \%$. Main experimental sources of systematics are summarized in Table 2.

The cross section is measured using a constrained fit on the number of observed events in all channels. A profile likelihood is used with nuisance parameters for each source of systematic uncertainties discussed in the previous paragraph. The resulting total $\mathrm{ZZ}$ production cross section, extrapolated to the full acceptance, is measured to :

$$
\sigma(\mathrm{pp} \rightarrow \mathrm{ZZ}+\mathrm{X})=3.8_{-1.2}^{+1.5} \text { (stat.) } \pm 0.2 \text { (syst.) } \pm 0.2 \text { (lumi.) } \mathrm{pb} .
$$

This cross section is consistent with the standard model prediction of $6.4 \pm 0.6 \mathrm{pb}$.

\section{Measurement of $\mathrm{WZ}$ production cross section}

Only leptonic final states involving electrons and muons are used to measure the WZ production cross section. Final state containing $\tau$ are not considered as signal. Contamination from $\tau$ decays are estimated to $6 \%$ of the total yield using a PYTHIA simulation. This contamination is subtracted in the cross section calculation. Events of interest are characterized by two same-flavor isolated leptons of opposite sign $\left(p_{T}>20(10) \mathrm{GeV}\right.$ for the electron channel and $p_{T}>15(15) \mathrm{GeV}$ for the muon channel) with an invariant mass in the range $[60-120] \mathrm{GeV}$. An additionnal isolated lepton $\left(p_{T}>20 \mathrm{GeV}\right)$ and a significant missing transverse energy $\left(E_{T}^{\text {miss }}>30 \mathrm{GeV}\right)$ associated to the neutrino produced in the $\mathrm{W}$ boson decay are also required. The invariant mass spectrum of the $\mathrm{Z}$ boson candidate is illustrated of Fig. 3. Lepton-combinatorial ambiguities are solved by taking the $\mathrm{Z}$ boson candidate with mass closest to the nominal $\mathrm{Z}$ boson mass.

$\mathrm{Z}+$ jets and $t \bar{t}$ backgrounds are estimated from data, using the "matrix method", based on the knowledge of the lepton selection efficiency and the probability that a jet fakes a lepton. These two numbers are computed on dedicated control samples: The "tag and probe" method on the inclusive $\mathrm{Z}$ sample is used to estimate the lepton selection efficiency ; a $\mathrm{Z}+$ jets sample is exploited to extract the lepton fake rate. We estimate the contamination of the main sample by fake leptons to 8.1 events for all channels. Uncertainty on WZ yield due to the background subtraction depends on the WZ decay channel and are contained in the range $2.9-4.9 \%$. Other backgrounds, $\mathrm{ZZ} \rightarrow 4 \ell$ and $\mathrm{Z} \gamma$, are small and are estimated from simulation to about one event. A systematic uncertainty of $20 \%$ is assigned to these backgrounds, leading to an overall uncertainty smaller than $1.7 \%$ on WZ yield. 


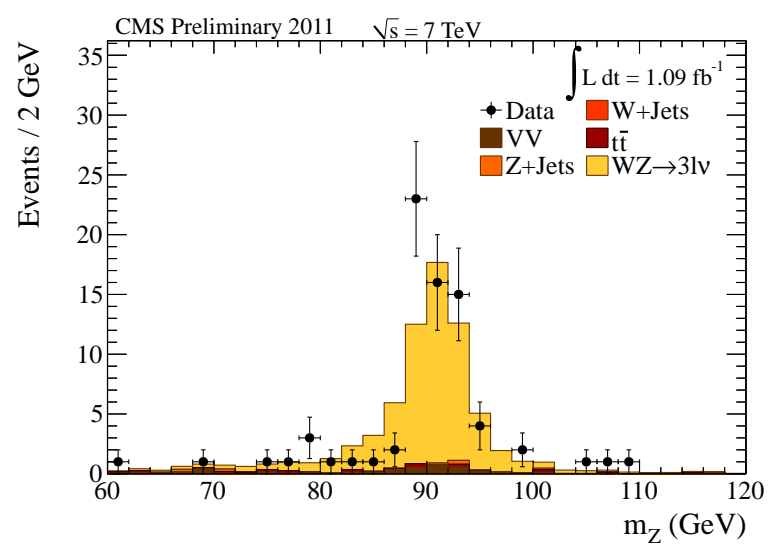

Figure 1: Invariant mass of the $\mathrm{Z}$ system in the WZ analysis, for data (black dots) and simulation (colored areas). 75 events are selected.

\begin{tabular}{c|c|c}
\hline \hline channel & $N_{\text {observed }}$ & cross section $(\mathrm{pb})$ \\
\hline$\sigma_{W Z \rightarrow e e e v}$ & 22 & $0.086 \pm 0.022($ stat $) \pm 0.007($ syst $) \pm 0.005($ lumi $)$ \\
$\sigma_{W Z \rightarrow e e \mu v}$ & 20 & $0.060 \pm 0.017($ stat $) \pm 0.005($ syst $) \pm 0.004($ lumi $)$ \\
$\sigma_{W Z \rightarrow \mu \mu e v}$ & 13 & $0.053 \pm 0.018($ stat $) \pm 0.004($ syst $) \pm 0.003($ lumi $)$ \\
$\sigma_{W Z \rightarrow \mu \mu \mu v}$ & 20 & $0.060 \pm 0.016($ stat $) \pm 0.004($ syst $) \pm 0.004($ lumi $)$ \\
\hline \hline
\end{tabular}

Table 3: Observed WZ candidate events and cross sections for $\int \mathscr{L} d t=1.1 \mathrm{fb}^{-1}$ per channel

Selection efficiencies are obtained from simulation and corrected using a inclusive $\mathrm{Z}$ control sample. Bounds on systematic uncertainties linked to selection efficiencies are $4.5-7.5 \%$, depending on the decay channel.

The total cross section, extrapolated to full detector acceptance, is computed for each decay channel. The results are consistent across channels (Tab. 3). Channels are combined and the WZ production cross section is estimated to :

$$
\sigma(\mathrm{pp} \rightarrow \mathrm{WZ}+\mathrm{X})=17.0 \pm 2.4 \text { (stat.) } \pm 1.1 \text { (syst.) } \pm 1.0(\text { lumi. }) \mathrm{pb}
$$

consistent with the standard model prediction.

\section{Measurement of $W W$ production cross section}

WW events in leptonic decays (no $\tau$ decay) are identified by the presence of two isolated leptons of high transverse momentum, $p_{T}>20(10) \mathrm{GeV}$, and a significant missing transverse energy coming from the two neutrinos. The $E_{T}^{\text {miss }}$ variable is built with the $E_{T}^{\text {miss }}$ projection along the transverse axis of the nearest lepton to reject $\mathrm{Z} \rightarrow \tau \tau$ background and a treatment against pile-up is made. Only events with $E_{T}^{\text {miss }}>40(20) \mathrm{GeV}$ for $e e / \mu \mu(e \mu)$ final state are selected.

Backgrounds are copious come from multiple sources. A jet veto at $30 \mathrm{GeV}$ and a b-tagging veto are used to reject top and $\mathrm{Z}+$ jets events. Other diboson events are rejected by applying a lepton veto. The Drell-Yan background is only present for same-flavor final states and is reduced by applying a veto on the di-lepton invariant mass window [76-106] GeV, rejecting events with invariant mass below $12 \mathrm{GeV}$ and requiring that the remaining jet and the di-lepton system are not emitted back-to-back in the transverse plane. The invariant mass spectrum of selected events is 


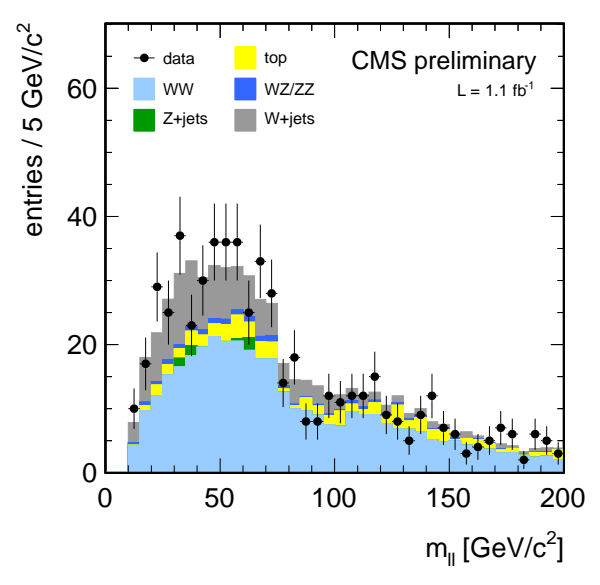

\begin{tabular}{c|c}
\hline \hline Sample & yield \\
\hline $\mathrm{qq} \rightarrow \mathrm{WW}$ & $349.7 \pm 30.3$ \\
$\mathrm{gg} \rightarrow \mathrm{WW}$ & $17.2 \pm 1.6$ \\
\hline $\mathrm{Z}+$ jets & $106.9 \pm 38.9$ \\
top & $63.8 \pm 15.9$ \\
resonnant $\mathrm{Z}+\mathrm{X} / \mathrm{Z} / \mathrm{W}$ & $12.2 \pm 5.3$ \\
$\mathrm{Z} \rightarrow \tau \tau$ & $1.6 \pm 0.4$ \\
non resonnant WZ/ZZ & $8.5 \pm 0.9$ \\
$\mathrm{~W} \gamma$ & $1.6 \pm 0.4$ \\
\hline Signal+background & $568.6 \pm 52.2$ \\
\hline \hline Data & 626 \\
\hline \hline
\end{tabular}

Table 4: Predicted signal and background yields, with the number of event selected in data.
Figure 2: Invariant mass of the di-lepton system of WW candidates, for data (black dots) and simulation (colored areas). All final states are combined.

Table 5: Main sources of systematic uncertainties for the WW cross section measurement in 2011 made by CMS. The uncertainties on background estimation are relative to background yields.

presented on Fig. 4. Backgrounds are estimated using data driven methods for the dominant backgrounds : fake leptons backgrounds $(\mathrm{QCD} / \mathrm{Z}+$ jets), top background and backgrounds containing one $\mathrm{Z}$ resonnance. Other backgrounds are smaller (non-resonnant $\mathrm{Z}, \mathrm{Z} \rightarrow \tau \tau$ and $\mathrm{W} \gamma$ processes) and are estimated with the simulation. Total number of selected events, signal prediction and background predictions are given in Table 4. Systematics are not given in detail but main sources and effects are given in Table 5.

Using 2011 data, the total WW production cross section is estimated to

$$
\sigma(\mathrm{pp} \rightarrow \mathrm{WW}+\mathrm{X})=55.3 \pm 3.3 \text { (stat.) } \pm 6.9 \text { (syst.) } \pm 3.3 \text { (lumi.) } \mathrm{pb}
$$

consistent with the Standard Model prediction of $43.0 \mathrm{pb}$ and with an earlier measurement made with 2010 data :

$$
\sigma(\mathrm{pp} \rightarrow \mathrm{WW}+\mathrm{X})=41.1 \pm 15.3 \text { (stat.) } \pm 5.8 \text { (syst.) } \pm 4.5 \text { (lumi.) pb. }
$$

\section{Measurement of $\mathbf{Z} \gamma$ and $\mathbf{W} \gamma$ production cross section}

$\mathrm{Z} \gamma$ and $\mathrm{W} \gamma$ analyses were performed with 2010 data and presented at Moriond 2011 (cf. [5,6]). 


\begin{tabular}{|c|c|c|c|}
\hline \hline WW $\gamma$ & $\mathrm{ZZ} \gamma$ & $\mathrm{Z} \gamma \gamma$ & WWZ \\
\hline$-0.61<\Delta \kappa_{\gamma}<0.65$ & $-0.05<h_{3}^{\gamma}<0.06$ & $-0.07<h_{3}^{Z}<0.07$ & $-0.29<\Delta g_{1}^{Z}<0.31$ \\
$-0.18<\lambda_{\gamma}<0.17$ & $-0.0005<h_{4}^{\gamma}<0.0005$ & $-0.0005<h_{4}^{Z}<0.0006$ & $-0.23<\lambda_{Z}<0.23$ \\
\hline \hline
\end{tabular}

Table 6: Limits on anomalous triple gauge couplings set by CMS using 2010 data $\left(36 \mathrm{pb}^{-1}\right)$. No form factors were used in the modelling of anomalous triple gauge couplings.

A brief description of these analyses and results are presented here. $\mathrm{Z}$ bosons are selected by requiring two isolated leptons with $p_{T}>20 \mathrm{GeV}$. Only reconstructed $\mathrm{Z}$ bosons with an invariant mass greater than $50 \mathrm{GeV}$ are used. W bosons are reconstructed with an isolated lepton with $p_{T}>20 \mathrm{GeV}$ and a missing transverse energy greater than $25 \mathrm{GeV}$. For both analyses, photons must have $p_{T}>10 \mathrm{GeV}$ and must respect the condition $\Delta R(\gamma$, lepton $)=\sqrt{\Delta \eta^{2}+\Delta \phi^{2}}>0.7$. These requirements define also the phase space used for cross section measurements, giving the following results :

$$
\begin{gathered}
\sigma(\mathrm{pp} \rightarrow \mathrm{Z} \gamma+X) \times \mathscr{B}(\mathrm{Z} \rightarrow \ell \ell)=9.4 \pm 1.0(\text { stat. }) \pm 0.6(\text { syst. }) \pm 0.4 \text { (lumi.) } \mathrm{pb} \\
\sigma(\mathrm{pp} \rightarrow \mathrm{W} \gamma+X) \times \mathscr{B}(\mathrm{W} \rightarrow \ell v)=56.3 \pm 5.0 \text { (stat. }) \pm 5.0 \text { (syst.) } \pm 2.3 \text { (lumi. }) \mathrm{pb}
\end{gathered}
$$

\section{Search for anomalous triple gauge couplings}

Limits on anomalous triple gauge couplings have been set using results based on 2010 data only. The corresponding deviation is modelled by an effective Lagrangien built without any form factor and requiring the respect of $\mathrm{SU}(2) \times \mathrm{U}(1)$ gauge invariance and $\mathrm{C}$ and $\mathrm{P}$ symmetries. From $\mathrm{W} \gamma$ and $\mathrm{Z} \gamma$ analyses are extracted limits on $\Delta \kappa_{\gamma}$ and $\lambda_{\gamma}\left(=\lambda_{\mathrm{Z}}\right)$, couplings associated to the $\mathrm{WW} \gamma$ vertex and on $h_{i}^{\gamma}, h_{i}^{Z}(i=3,4)$ related to the $\mathrm{ZZ} \gamma$ and $\mathrm{Z} \gamma \gamma$ vertices. The WW analysis permits to constrain the $\lambda_{Z}, \kappa_{\gamma}$ and $g_{1}^{Z}$ couplings.

All limits are set using unbinned fits on photon (in $\mathrm{W} \gamma$ and $\mathrm{Z} \gamma$ events) or leading lepton (WW events) $p_{T}$ spectra. Sensitivity is found to be similar to that of Tevatron experiments. The $95 \%$ C.L. limits on deviations from SM prediction are summarized in Table 6.

\section{References}

[1] The CMS collaboration, Measurement of WW and observation of WZ and ZZ in leptonic modes, CMS-PAS-EWK-11-010, 2011.

[2] The CMS collaboration, Measurement of Wr and $Z \gamma$ production in pp collisions at $\sqrt{s}=7$ TeV, Phys. Lett. B701, 535-555 (2011). doi:10.1016/j.physletb.2011.06.034

[3] The CMS collaboration, Measurement of $W^{+} W^{-}$production and search for Higgs boson in pp collision at $\sqrt{s}=7 \mathrm{TeV}$, Phys. Lett. B699, 25-47 (2011). doi:10.1016/j.physletb.2011.03.96

[4] The CMS collaboration, The CMS experiment at the CERN LHC, JINST 3(2008) S08004. doi:10.1088/1748-0221/3/08/S08004.

[5] P. Harris, Standard Model measurements, CMS-CR-11-068, 2011.

[6] D. Majumder, Study of Wr events at the CMS with 7 TeV LHC data, CMS-CR-11-076, 2011. 